\title{
Management of group b streptococcus- positive pregnant women at maternity homes in JAPAN: a questionnaire survey of compliance among midwives
}

Kotomi Yamaguchi ${ }^{1,2^{*}}$ and Kazutomo Ohashi ${ }^{3}$

\begin{abstract}
Background: Per the 2014 Japanese Midwives Association (JMA) guidelines, midwives were allowed to manage the deliveries for group B streptococcus (GBS)-positive pregnant women in labour at maternity homes without the supervision of a medical doctor if they complied with the guidelines of the Japan Society of Obstetrics and Gynecology (JSOG), wherein midwives working for maternity homes are expected to cooperate with commissioned obstetricians and paediatricians in cooperative medical facilities. We examined the rate of compliance with these JMA and JSOG guidelines regarding the management of GBS-positive pregnant women among midwives at maternity homes in Japan.
\end{abstract}

Methods: Between October and December 2015, an anonymous questionnaire was distributed to 337 maternity homes registered with the JMA by mail. The questionnaire obtained information regarding the timing of GBS screening, specimen collection, transfer of GBS-positive pregnant women from a maternity home to a hospital, administration of intrapartum antibiotic prophylaxis, and collaboration between midwives and commissioned obstetricians. Data were analysed using descriptive statistics. We used frequency distribution as the statistical test.

Results: Responses were received from 246 (73.0\%) maternity homes, of which complete responses from 204 maternity homes (valid response rate, 60.5\%) were analysed. Of these 204 maternity homes, only 97 (47.5\%) conducted a GBS screening test during 33-37 weeks of gestation as recommended by the JSOG guidelines. Although midwives alone managed GBS-positive pregnant women in labour at 135 maternity homes (66.2\%), intrapartum antibiotic prophylaxis, as recommended by the JSOG guidelines, was conducted in only 111 (54.4\%). Moreover, only $37.0 \%$ (50/135) and $82.2 \%(111 / 135)$ of maternity homes ensured that GBS-positive pregnant women in labour with an elapse of $\geq 18 \mathrm{~h}$ after PROM and a body temperature of $\geq 38.0^{\circ} \mathrm{C}$, respectively, were transferred to a hospital by ambulance. Only at $58.3 \%$ (119/204) of maternity homes did midwives discuss the management of labour for GBS-positive pregnant women with commissioned obstetricians.

Conclusions: Some midwives working for maternity homes did not follow the JMA and JSOG guidelines of the management of GBS-positive pregnant women. For improving compliance rates, midwives at maternity homes should discuss the management of GBS-positive pregnant women with commissioned doctors more carefully and concretely per the existing guidelines.

Keywords: Group B streptococcus, Maternity home, Questionnaire survey, Japan, Screening, Guideline

\footnotetext{
* Correspondence: kotomi@gifu-u.ac.jp

'Department of Human Health Sciences, Kyoto University Graduate School of

Medicine, 53 Kawahara-chou, Shougoin, Sakyou-ku, Kyoto 606-8507, Japan

${ }^{2}$ Present Address: School of Medicine Nursing Course, Gifu University, 1-1

Yanagido, Gifu 505-1194, Japan

Full list of author information is available at the end of the article
} 


\section{Background}

About 20\% of pregnant women develop Group B streptococcus (GBS) colonization around their vagina and/or recto-anal region [1]. 64 infants of 118 colonized pregnant women who does not received intrapartum antibiotics at delivery were colonized with GBS, and the rate of vertical transmission of GBS was 52.5\% [2]. GBS causes severe infection such as sepsis, pneumonia, and meningitis in infants under 3 months old. Neonatal GBS infection can be divided into early-onset disease (EOD), occurring within 6 days after birth, and late-onset disease (LOD) occurring, 7 days or later after birth. In the absence of prophylactic treatment for GBS-positive pregnant women, $1-2 \%$ of newborns develop EOD by vertical transmission [3].

In 1996, the Centers for Disease Control and Prevention (CDC) recommended a universal screening program for GBS among pregnant women as well as intrapartum antibiotic prophylaxis for GBS-positive pregnant women to reduce the incidence of EOD. On the other hand, in the United Kingdom and New Zealand, a universal and routine antenatal GBS screening method was not recommended; instead, a risk-based GBS prevention strategy was employed $[4,5]$. In Japan, the guidelines of the Japan Society of Obstetrics and Gynecology (JSOG) were issued in 2008 following CDC recommendations, JSOG recommended to conduct the GBS screening test between 33 and 37 weeks of gestation in all pregnant women [6]. In a nationwide surveillance study investigated after the guidelines of JSOG, the mortality rates for EOD improved from $14.8 \%$ to $11.8 \%$. [7].

Midwives in Japan can manage normal and spontaneous deliveries without the supervision of an obstetrician; however, they cannot prescribe treatment, such as antibacterial agents, or conduct any tests without directions from the medical doctor. Midwives working at maternity homes can handle normal and spontaneous deliveries alone only in the presence of a contract that mandates the cooperation of obstetricians and paediatricians (hereafter, commissioned obstetricians/ paediatricians/medical doctors) from medical institutes. Maternity homes see approximately 10,000 births per year ( $0.9 \%$ of the total number of deliveries in 2014). Before 2014, cases of GBS-positive pregnant women going into labour were considered as requiring special attention, such that midwives working at maternity homes would not manage these deliveries without the supervision of an obstetrician. In 2014, the Japanese Midwives Association [8] issued new guidelines according to which midwives working at maternity homes were allowed to handle the deliveries for GBS-positive pregnant women only if they comply with the guidelines of the JSOG and promote cooperation with commissioned obstetricians and paediatricians. However, whether the midwives actually comply with these guidelines is not known.

\section{Objectives}

The aim of this study was to examine the status of compliance with the current guidelines (issued by the Japanese Midwives Association [JMA] and JSOG) for managing GBS-positive pregnant women among midwives working at maternity homes in Japan.

\section{Methods \\ Study design}

A cross-sectional study design was used. Ethics approval and consent to participate.

The Ethical Committee of Kyoto University Medical School approved the study (Approval number R0153). Responses to the questionnaire from the maternity homes were considered informed consent. The anonymity of the participants was preserved.

\section{Setting}

Maternity homes in Japan which deal with delivery.

\section{Participants}

We sent an anonymous self-administered questionnaire and an informed consent form to 337 maternity homes registered on the JMA website by mail between September and December 2015. The questionnaire obtained information regarding the numbers of midwives and births per year at each maternity home, and the status of compliance with guidelines regarding GBS-positive pregnant women in terms of (1) the timing of the GBS screening test, (2) the person in charge of specimen collection, (3) the management of GBS-positive pregnant women in labour including transfer from a maternity home to a hospital and administration of intrapartum antibiotic prophylaxis, and (4) the discussion of these issues between midwives and commissioned obstetricians.

Recommendations for managing pregnant women with a GBS infection per the JSOG guideline.

1. Conduct the GBS screening test between 33 and 37 weeks of gestation.

2. Collect a specimen for GBS culture via a lower vaginal and anal canal swab.

3. Administer prophylactic antibiotic treatment to GBS-positive women who are in labour and scheduled to give birth via vaginal delivery or after premature rupture of membranes during pregnancy.

\section{Date sources}

JSOG guidelines

Recommendations for managing pregnant women with a GBS infection per the JMA guideline. 
Midwives can manage the delivery for GBS-positive pregnant women at maternity homes without the supervision of the obstetrician only if they comply with the guidelines of the JSOG and cooperate with the commissioned obstetricians to prepare for unforeseeable accidents. Moreover, GBS-positive pregnant women in labour should be taken to a hospital via ambulance in the following cases: elapse of $\geq 18 \mathrm{~h}$ after the premature rupture of membranes (PROM) and maternal body temperature of $\geq 38.0^{\circ} \mathrm{C}$.

\section{Statistical methods}

The descriptive statistical analyses were carried out with the IBM SPSS Statistics 21.0. (IBM Japan Ltd., Tokyo, Japan). We used frequency distribution as the statistical test.

\section{Results}

\section{Participants}

We received responses to the questionnaire survey from 246 (73.0\%) maternity homes and analysed complete responses from 204 maternity homes (valid response rate, $60.5 \%$ ). Forty-two responses from 32 maternity homes that do not deal with labour and 10 that did not want to participate in the study were excluded.

Overall, maternity homes employed 1-14 midwives, and the most frequent number of midwives employed was 2 , in $38.1 \%$ of maternity homes. Moreover, $22.8 \%$ of maternity homes employed 1 midwife and $16.3 \%$ employed 3 midwives. The number of deliveries in 2014 ranged 0-208, and the most frequent number of deliveries was 15 , which was reported for $5.4 \%$ of maternity homes. The median number of deliveries was 16 , and $20.9 \%$ of maternity homes reported managing fewer than 5 deliveries in a year.

\section{Main results}

\section{Timing of screening and specimen collection}

GBS screening tests were conducted twice in 3 (1.5\%) maternity homes, and the timing of the second test as used for statistical analysis. Medical professionals in 97 (47.5\%) maternity homes conducted the GBS screening test during 33-37 weeks of gestation as recommended by the JSOG guideline. Medical professionals in charge of specimen collection were as follows: commissioned obstetricians in $194(95.1 \%)$ cases, midwives in $6(2.9 \%)$, obstetricians or midwives in 3 (1.5\%), and a clinical laboratory technician in $1(0.5 \%)$ (Table 1$)$.

\section{Managing GBS-positive pregnant women in labour}

Of the 135 (66.2\%) maternity homes wherein midwives handled GBS-positive pregnant women in labour, 111 $(82.2 \%)$ reported the use of intrapartum antibiotic prophylaxis. Five maternity homes that responded "others" indicated the use of oral antibiotics. Moreover, only 37.0\% $(50 / 135)$ and $82.2 \%(111 / 135)$ of maternity homes ensured that GBS-positive pregnant women in labour with an elapse of $\geq 18 \mathrm{~h}$ after PROM and a body temperature of $\geq 38.0{ }^{\circ} \mathrm{C}$, respectively, were transferred to a hospital by ambulance (Table 2).

\section{Collaboration between midwives and commission obstetricians}

In only $58.3 \%(119 / 204)$ of maternity homes did the midwives and commissioned obstetricians discuss the management of GBS-positive pregnant women in labour. In $63(30.9 \%)$ maternity homes, the discussion did not take place, and relevant responses were not obtained from 8 (3.9\%) maternity homes. Lastly, 9 of 14 maternity homes that provided a response of "others" claimed that discussion for JSOG guidelines regarding the handling of GBS-positive pregnant women in labour at maternity homes was in place before the JMA guidelines were revised in 2014, which midwives could deal with GBG positive pregnant women at maternity homes.

\section{Discussions}

\section{Key results}

In compliance with the JSOG guidelines, only $47.5 \%$ of maternity homes reported conducting GBS screening within the recommended period. This percentage was lower than the $64.4 \%$ of certified nurse midwives [9] and 97.1\% of obstetricians [10] who did comply with the relevant guidelines in the United States. Nevertheless, the recommended timing for screening differs between

Table 1 Timing of GBS screening for pregnant women

\begin{tabular}{|c|c|c|c|c|c|c|c|c|c|c|c|}
\hline gestational weeks & $202(\%)$ & $\sim 32 \quad 33$ & 34 & 35 & 36 & 37 & 38 & 39 & 40 & 41 & 42 \\
\hline$\sim 32$ weeks 6 days & $15(7.4)$ & & & & & & & & & & \\
\hline$\sim 37$ weeks 6 days & $53(26.0)$ & & & & & & & & & & \\
\hline 33 weeks 0 days $\sim 37$ weeks 6 days & $97(47.5)$ & & & & & & & & & & \\
\hline 33 weeks 0 days & $32(15.7)$ & & & & & & & & & & \\
\hline all weeks & $1(0.5)$ & & & & & & & & & & \\
\hline 38 weeks 0 days $~$ & $4(2.0)$ & & & & & & & & & & \\
\hline
\end{tabular}


Table 2 Management for GBS-positive pregnant women

\begin{tabular}{ll}
\hline Manegement & $n=135(\%)$ \\
\hline Antibiotic prophylaxis during delivery & $111(82.2 \%)$ \\
No & $16(11.9 \%)$ \\
Others & $6(4.4 \%)$ \\
No answer & $2(1.5 \%)$ \\
Ambulance transport in case of $\geq 18$ h after the rupture of the \\
membranes \\
Yes & $50(37.0 \%)$ \\
No & $32(23.7 \%)$ \\
Others & $51(37.8 \%)$ \\
No answer & $2(1.5 \%)$ \\
Ambulance transport in case of maternal body temperature of $\leq 38.0^{\circ} \mathrm{C}$ \\
Yes & $111(82.2 \%)$ \\
No & $1(0.7 \%)$ \\
Others & $20(14.8 \%)$ \\
No answer & $3(2.2 \%)$ \\
\hline
\end{tabular}

the JSOG (33-37 weeks of gestation) and CDC guidelines (35-37 weeks of gestation). Although the time window for the test is longer in Japan than in the United States, the compliance rate was lower in midwives working at maternity homes in Japan. In Germany, only 59.3\% of pregnant women visiting a tertiary medical care centre underwent a GBS screening test at 35-37 gestational weeks, and the reason for this low compliance rate was reported as the GBS screening test not being covered by medical insurance in Germany [11]. However, the GBS screening test in Japan is covered by the government insurance, which does not explain the low compliance rate observed in Japan.

Additionally, $82.2 \%$ of midwives received intrapartum antibiotic prophylaxis, and this percentage was lower than that for certified nurse midwives in the United States (92.5\%) as well [9]. In this study, the midwives received prescriptions for oral medicines from the commissioned medical doctors for GBS-positive pregnant women. A safe and efficacious vaccine has not achieved licensure thus far $[3,12,13]$, which makes the adequate use of antibiotics essential for the prevention of mother-to-child infection. There is no recommendation regarding the appropriate time for administration of intrapartum antibiotic prophylaxis in the JSOG and JMA guidelines. A previous study conducted in Japan reported that some infants born to GBS-positive women who received antibiotic prophylaxis within $4 \mathrm{~h}$ before delivery developed EOD [14], and GBS was detected in these infants [15]. In 2002, the CDC revised the guidelines for intrapartum antibiotic prophylaxis, wherein antibiotics administered at $\geq 4$ h before delivery and any other administrations of antibiotics during pregnancy and labour were deemed unnecessary [3]. However, obstetricians and midwives in the United States were extremely concerned about the timely administration of antibiotics [16]. These results suggest that midwives working at maternity homes in Japan may not be aware of the consequences of GBS infection prophylaxis, find these procedures rather troublesome, or do not ensure adequate collaboration with the commissioned medical doctors. Midwives in Japan cannot conduct any clinical tests or prescribe medicines themselves; therefore, they need to cooperate with commissioned obstetricians in the management of GBS-positive pregnant women.

The CDC provided the algorithm for the secondary prevention of GBS infection in infants and recommended "limited evaluation" including a blood culture at birth, a complete blood count including a differential white blood cell and platelet count at birth and/or 6$12 \mathrm{~h}$ after birth, as well as antibiotic administration for infants born to GBS-positive mothers with chorioamnionitis [3]. In cases of inadequate intrapartum antibiotic prophylaxis for GBS-positive pregnant women at $\geq 18 \mathrm{~h}$ after PROM, "limited evaluation" and additional observation for $\geq 48 \mathrm{~h}$ is recommended. The Committee on the Fetus and Newborn also recommended that infants born to GBS-positive mothers $\geq 18 \mathrm{~h}$ after ROM should undergo a blood test $[17,18]$. Most cases of EOD demonstrate an acute onset within $24 \mathrm{~h}$, and its prognosis is severe [5, 19]; therefore, early detection and treatment are important. Although the JSOG guidelines do not mention secondary prevention, midwives working at maternity homes should ensure the women's transfer to a hospital by ambulance when necessary according to the JAM recommendations. In the present study, the rate of compliance with this guideline was very low (37.0\%) and some midwives answered that they made arbitrary decisions in cases of $24 \mathrm{~h}$ since PROM or consulted a doctor about hospital transfer each time. Moreover, only $82.2 \%$ of midwives referred pregnant women in labour to a hospital by ambulance even though fever is an obvious sign of septic infection due to GBS [20, 21]. In such cases, there is a high possibility of a delay in the primary treatment for EOD.

Thus, the compliance with these two guidelines needs to be improved quickly through discussion with commissioned obstetricians. However, only $58.3 \%$ of midwives reported discussing the management of GBS-positive pregnant women in labour with commissioned obstetricians. It's a quite previous study, but it reported that $44 \%$ of obstetricians and $39 \%$ of neonatologists did not support universal antenatal screening in Australia. [22]. In statement for GBS of Royal Australian and New Zealand College of Obstetricians and Gynaecologists recommended either a risk-based approach or culturebased screening, and midwives at homebirth could not 
deal with delivery for GBS positive pregnant women or those who not undergo culture-based screening [23]. On the basis of the current findings, we can expect the same situation in Japan. It is important to understand the outcomes of the collaboration between midwives and medical doctors in Japan. Pregnant women have the right to receive adequate treatment and care at any institution, and midwives must therefore comply with the guidelines that recommend cooperation with commissioned doctors. To improve the compliance rate among midwives working at maternity homes, it is also necessary to understand why they do not comply with the recommended guidelines and thus promote their consciousness of risk management.

\section{Conclusions}

In conclusion, more than half of the maternity homes investigated did not conduct the GBS screening test within the gestational period recommended by the JSOG guideline. Despite introducing the new JMA guidelines according to which midwives could manage deliveries for GBS-positive pregnant women at maternity homes themselves only if they complied with the JSOG guideline and cooperate with commissioned doctors, only $58.3 \%$ of midwives discussed the management of GBSpositive pregnant women with commissioned obstetricians. Although $66.2 \%$ of maternity homes managed GBS-positive pregnant women in labour, only $37.0 \%$ ensured transfer of these pregnant women to a hospital in case of $\geq 18 \mathrm{~h}$ had elapsed since PROM. Additional education and promoting discussion with obstetricians for midwives would be essential to improve the current compliance rate.

\section{Limitations}

A limitation of this study should be acknowledged. We included 337 maternity homes opened on the JMA website based on personal information protection out of 441 maternity homes in Japan. Although the response rate was high $(73.0 \%)$, we conducted this survey within 1 year after the JMA guidelines were revised. Therefore, there is a possibility that midwives and commissioned doctors might not be very familiar with the revised guidelines. Thus, the status of compliance needs to be evaluated continually. Despite this limitation, this study represents the first investigation of compliance with GBS infection-related guidelines among midwives working at maternity homes, and provides useful information in terms of the scope for improvement in the current approach to preventing vertical transmission of GBS.

\section{Interpretation}

The license of obstetrician and midwives are different in each country. Therefore it is necessary for each other to cooperate so that they can conduct evidence-based medical practice in different countries.

\section{Abbreviations}

CDC: Centers for Disease Control and Prevention; EOD: Early-onset disease; GBS: Group B streptococcus; JMA: Japanese Midwives Association;

JSOG: Japan Society of Obstetrics and Gynecology; LOD: Late-onset disease; PROM: Premature rupture of membranes

\section{Acknowledgements}

The authors sincerely thank the midwives who participated in our study.

\section{Funding}

JSPS KAKENHI Grant Number 16K12141 and Kyoto University Graduate School of Medicine provided funding and resources for this research. The authors declare that they have no competing interest.

\section{Availability of data and materials}

The datasets used and analysed during the current study are available from the corresponding author on reasonable request.

\section{Authors' contributions}

KY conceptualized and designed the study; collected, analysed, and interpreted the data; and drafted the manuscript. KO conceptualized and designed the study and drafted the manuscript. Both authors approve the final version of the manuscript.

\section{Ethics approval and consent to participate}

The Ethical Committee of Kyoto University Medical School approved the study (Approval number R0153). Responses to the questionnaire from the maternity homes were considered informed consent. The anonymity of the participants was preserved.

\section{Consent for publication}

Not applicable

\section{Competing interests}

The authors declare that they have no competing interest.

\section{Publisher's Note}

Springer Nature remains neutral with regard to jurisdictional claims in published maps and institutional affiliations.

\section{Author details}

${ }^{1}$ Department of Human Health Sciences, Kyoto University Graduate School of Medicine, 53 Kawahara-chou, Shougoin, Sakyou-ku, Kyoto 606-8507, Japan. ${ }^{2}$ Present Address: School of Medicine Nursing Course, Gifu University, 1-1 Yanagido, Gifu 505-1194, Japan. 3Department of Children and Women's Health, Division of Health Sciences, Osaka University Graduate School of Medicine, 1-7 Yamada-oka, Suita, Osaka 565-0871, Japan.

Received: 28 August 2017 Accepted: 5 December 2017

Published online: 05 February 2018

References

1. Kwatra G, Cunnington MC, Merrall E, Adrian PV, Ip M, Klugman KP, et al. Prevalence of maternal colonisation with group B streptococcus: a systematic review and meta-analysis. Lancet Infect Dis. 2016;16(9):1076-84

2. Hickman ME, Rench MA, Ferrieri $P$, Baker CJ. Changing epidemiology of group B streptococcal colonization. Pediatrics. 1999;104(2):203-9.

3. Centers for Disease Control and Prevention (2010). Prevention of Perinatal Group B Streptococcal Disease Revised Guideline from CDC, 2010. https:// www.cdc.gov/groupbstrep/guidelines/guidelines.html. Accessed 3 April 2016.

4. National Institute for Health and Care Excellence (2015). Xpert GBS test for the intrapartum detection of group B streptococcus. NICE advice (MIB28). https://www.nice.org.uk/advice/mib28. Accessed 3 April 2016.

5. Darlow B, Campbell N, Austin N, Chin A, Grigg C, Skidmore C, et al. The prevention of early-onset neonatal group $B$ streptococcus infection: New Zealand consensus guidelines 2014. N Z Med J. 2015;128(1425):69-76. 
6. Japan Society of Obstetrics and Gynecology and Japan Association of Obstetricians and Gynecologists. GBS hokinshindan to toriatsukai ha? In: Guidelines for Obstetric and gynecologic practice-obstetrics 2014. Tokyo: Japan Society of Obstetrics and Gynecology; 2014. p. 295-297 (in Japanese).

7. Matsubara K, Hoshina K, Suzuki Y. Early-onset and late-onset group B streptococcal disease in Japan: a nationwide surveillance study, 2004-2010. Int J Infect Dis. 2013;17:e379-84.

8. Japanese Midwives Association. In: Guidelines for Midwives practice. 2014. Tokyo: Japanese Midwives Association.16-17, 22, 45. (in Japanese).

9. Bloom KC, Ewing CA, Group B. Streptococcal (GBS) disease screening and treatment during pregnancy: nurse-midwives' consistency with 1996 CDC recommendations. J Midwifery Womens Health. 2001;46(1):17-23.

10. Edwards RK, Tang Y, Raglan GB, Szychowski JM, Schulkin J, Schrag JS. Survey of American obstetricians regarding group B streptococcus: opinions and practice patterns. Am J Obstet Gynecol. 2015;213(229):e1-7.

11. Kunze $M$, Zumstein $K$, Markfeld-Erol F, Elling R, Lander F, Prömpeler $H$, et al. Comparison of pre- and intrapartum screening of group B streptococci and adherence to screening guidelines: a cohort study. Eur J Pediatr. 2015;174(6):827-35.

12. Heath PT. Status of vaccine research and development of vaccines for GBS. Vaccine. 2016;34(26):2876-9.

13. Madhi SA, Dangor Z. Prospects for preventing infant invasive GBS disease through maternal vaccination. Vaccine. 2017; https://doi.org/10.1016/j. vaccine.2017.02.025.

14. Kojima K, Tanaka R, Nakajima K, Kurihara N, Oba MS, Yamashita Y, et al. Predicting outcomes of neonates born to GBS-positive women who received inadequate intrapartum antimicrobial prophylaxis. Turk J Pediatr. 2014;56(3):238-42.

15. Sakata H. Evaluation of intrapartum antibiotic prophylaxis for the prevention of early-onset group B streptococcal infection. J Infect Chemother. 2012; 18(6):853-7.

16. Barber EL, Funai EF, Bracken MB, Illuzzi JL. Interpretation of 2002 centers for disease control guidelines for group B streptococcus and evolving provider practice patterns. Am J Perinatol. 2011;28(2):97-102.

17. Polin RA. Committee on fetus and newborn. Management of neonates with suspected or proven early-onset bacterial sepsis. Pediatrics. 2012;129(5): 1006-15.

18. Tzialla C, Borghesi A, Longo S, Stronati M. Which is the optimal algorithm for the prevention of neonatal early-onset group B streptococcus sepsis? Early Hum Dev. 2014;90S1:35-8.

19. Matsubara K, Hoshina K, Kondo M, Miyairi I, Yukitake Y, Ito Y, et al. Group B streptococcal disease in infants in the first year of life: a nationwide surveillance study in Japan, 2011-2015. Infection. 2017; doi: https://doi.org/10.1007/s15010017-0995-2.

20. Clifford V, Garland SM, Grimwood K. Prevention of neonatal group B streptococcus disease in the 21st century. J Paediatr Child Health. 2012;48(9):808-15.

21. Miyata A, Takahashi H, Kubo T, Watanabe N, Tsukamoto K, Ito Y, et al. Earlyonset group B streptococcal disease following culture-based screening in Japan: a single center study. J Obstet Gynaecol Res. 2012:38(8):1052-6.

22. McLaughlin $\mathrm{K}$, Crowther $\mathrm{C}$. Universal antenatal group B streptococcus screening? The opinions of obstetricians and neonatologists within Australia. Aust N Z J Obstet Gynaecol. 2000;40(3):338-40.

23. Homer CS, Scarf V, Catling C, Davis D. Culture-based versus risk-based screening for the prevention of group B streptococcal disease in newborns: a review of national guidelines. Women Birth. 2014;27(1):46-51.

\section{Submit your next manuscript to BioMed Central and we will help you at every step:}

- We accept pre-submission inquiries

- Our selector tool helps you to find the most relevant journal

- We provide round the clock customer support

- Convenient online submission

- Thorough peer review

- Inclusion in PubMed and all major indexing services

- Maximum visibility for your research

Submit your manuscript at www.biomedcentral.com/submit
Biomed Central 